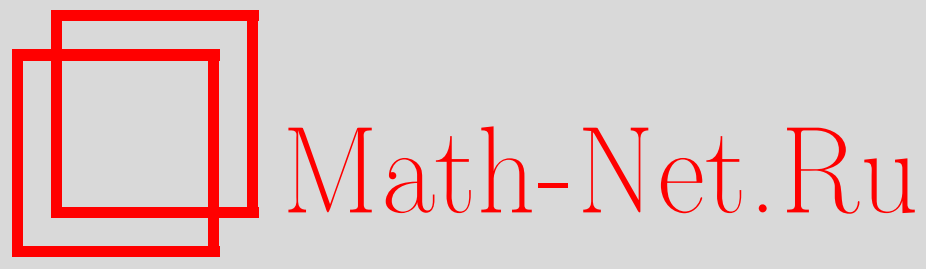

O. Hadjiliadis, V. H. Poor, On the Best 2-CUSUM Stopping Rule for Quickest Detection of Two-Sided Alternatives in a Brownian Motion Model, Теория вероятн. и ее примен., 2008, том 53, выпуск 3, 610-622

DOI: https://doi.org/10.4213/tvp2456

Использование Общероссийского математического портала Math-Net.Ru подразумевает, что вы прочитали и согласны с пользовательским соглашением

http://www.mathnet.ru/rus/agreement

Параметры загрузки:

IP: 54.80 .73 .141

26 апреля 2023 г., 13:15:54

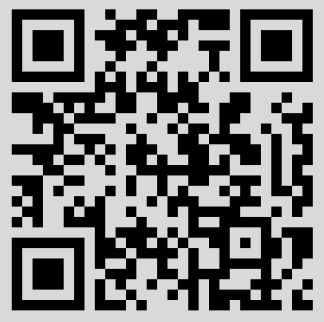




\title{
ON THE BEST 2-CUSUM STOPPING RULE FOR QUICKEST DETECTION OF TWO-SIDED ALTERNATIVES IN A BROWNIAN MOTION MODEL
}

\begin{abstract}
Изучается задача последовательного обнаружения изменения сноса броуновского движения в случае двусторонних альтернатив. Традиционно в этой задаче использовались двусторонние CUSUM-правила остановки ввиду их асимптотической оптимальности, когда среднее время между ложными тревогами стремится $\mathrm{K} \infty$. В частности, особое внимание привлекали двусторонние CUSUM-правила гармонического среднего - благодаря простоте вычисления их первых моментов. В настоящей статье получены выражения для первого момента обшего двустороннего CUSUM-правила остановки и скорости его изменения.

Эти выражения используются для вывода явных верхней и нижней оценок первого момента и скорости его изменения при изменении одного из пороговых параметров.

Используя эти выражения, мы доказываем не только существование, но и единственность наилучшего классического двустороннего CUSUM-правила остановки относительно обобщенного критерия Лордена, предложенного в [5]. В частности, мы описываем класс наилучших двусторонних CUSUM-правил остановки, как в симметричном, так и в несимметричном случае.
\end{abstract}

Ключевые слова и фразы: обнаружение изменений, наискорейшее обнаружение, кумулятивные суммы (CUSUM), двусторонние кумулятивные суммы.

1. Mathematical formulation. We sequentially observe a process $\left\{\xi_{t}\right\}$ with the following dynamics:

$$
d \xi_{t}=\left\{\begin{array}{ccc}
d w_{t}, & t<\theta \\
\mu_{1} d t+d w_{t} & \text { or } \quad-\mu_{2} d t+d w_{t}, & t \geqslant \theta
\end{array}\right.
$$

where $\theta$, the time of change, is assumed to be deterministic but unknown; $w_{t}$ is a standard Brownian motion process; $\mu_{i}$, the possible drifts to which the process can change, are assumed to be known, but the specific drift to

* Department of Mathematics, Brooklyn College, City University of New York, Brooklyn NY 11210, USA; e-mail: ohadjiliadis@gc.cuny.edu, ohadjiliadis@brooklyn.cuny.edu

** School of Engineering and Applied Science, Princeton University, C-230 Engineering Quadrangle, Olden Street, Princeton, NJ 08544, USA; e-mail: poor@princeton.edu 
which the process changes is unknown. Both $\mu_{1}$ and $\mu_{2}$ are assumed to be positive.

The probability triplet consists of $\left(C[0, \infty], \bigcup_{t>0} \mathscr{F}_{t}\right)$, where $\mathscr{F}_{t}=$ $\sigma\left\{\xi_{s}, 0<s \leqslant t\right\}$, and the families of probability measures $\left\{\mathscr{P}_{\theta}^{i}\right\}, \theta \in[0, \infty)$, whenever the change is $\mu_{i}, i=1,2$, at time $\theta$, and $\mathscr{P}_{\infty}$, the Wiener measure.

Our goal is to detect a change by means of a stopping rule $\tau$ adapted to the filtration $\left(\mathscr{F}_{t}\right)$. As a performance measure for this stopping rule we propose an extended Lorden criterion (see [5])

$$
J_{L}(\tau)=\max \left\{J_{1}(\tau), J_{2}(\tau)\right\},
$$

where $J_{i}(\tau)=\sup _{\theta} \operatorname{ess} \sup \mathbf{E}_{\theta}^{i}\left[(\tau-\theta)^{+} \mid \mathscr{F}_{\theta}\right], i=1,2$. This gives rise to the following min-max constrained optimization problem:

$$
\inf _{\tau} J_{L}(\tau) \quad \text { subject to } \quad \mathbf{E}_{\infty}[\tau] \geqslant T,
$$

where the constraint specifies the minimum allowable mean time between false alarms. As discussed in [7], in seeking solutions to the above problem, we can restrict our attention to stopping times that achieve the false alarm constraint with equality.

In the case of a one-sided change in this setting it has been shown that the one-sided CUSUM stopping rule is optimal (see [8] and [2]). The problem of detecting a change in the drift of a Brownian motion with two-sided alternatives is considerably more difficult than that with one-sided alternatives. This paper is a continuation of the work started in [5] and [4]. In the former it is conjectured but not proved that equalizer rules are best and two strong asymptotic optimality results as the mean time between false alarms tends to $\infty$ are presented both in the symmetric case, where a classical 2-CUSUM harmonic mean rule is proposed, and in the nonsymmetric case, where a modified drift 2-CUSUM harmonic mean equalizer rule is proposed. These asymptotic results enhance the 2-CUSUM asymptotic optimality results of [10]. In [4], it is seen that within the class of modified drift 2-CUSUM harmonic mean rules, the best rules occur when the drift parameters of the modified drift 2-CUSUM harmonic mean stopping rules $\lambda_{1}$ and $\lambda_{2}$ are chosen so that $\lambda_{2}-\lambda_{1}=2\left(\mu_{2}-\mu_{1}\right)$ (a relationship that results in an equalizer rule), for any value of the mean time between false alarms.

In this paper, we begin by a simple argument that demonstrates that the optimal stopping rule for problem (2) must satisfy $J_{1}(\tau)=J_{2}(\tau)$ and thus be an equalizer rule. This argument is summarized in the following two remarks.

$\mathrm{R}$ e $\mathrm{m}$ a r k 1 . Consider a stopping rule $\tau_{U}$ such that $J_{1}\left(\tau_{U}\right)>J_{2}\left(\tau_{U}\right)$ and let $\tau_{V}$ be a new stopping rule that declares an alarm at exactly the same instant as $\tau_{U}$ if and only if at each instant $t$ it receives the observations $\left\{-\xi_{s}\right.$, $s \leqslant t\}$. Then $J_{2}\left(\tau_{V}\right)>J_{1}\left(\tau_{V}\right)$ with $J_{1}\left(\tau_{U}\right)=J_{2}\left(\tau_{V}\right)$ and $J_{2}\left(\tau_{U}\right)=J_{1}\left(\tau_{V}\right)$. Now construct $\tau=\frac{1}{2} \tau_{U}+\frac{1}{2} \tau_{V}$. It is clear that for this stopping rule $J_{L}(\tau)<$ 
$J_{L}\left(\tau_{U}\right)=J_{L}\left(\tau_{V}\right)$ and $\mathbf{E}_{\infty}[\tau]=\mathbf{E}_{\infty}\left[\tau_{U}\right]=\mathbf{E}_{\infty}\left[\tau_{V}\right]$, while $J_{1}(\tau)=J_{2}(\tau)$. That is, we have found another stopping rule $\tau$ that, for the same mean time between false alarms, has achieved a lesser detection delay.

$\mathrm{R} \mathrm{e} \mathrm{mark} \mathrm{2.} \mathrm{It} \mathrm{is} \mathrm{important} \mathrm{to} \mathrm{point} \mathrm{out} \mathrm{that} \tau$ of Remark 1 is not technically a stopping rule with respect to $\mathscr{F}_{t}$, since randomization is involved in its construction. In other words, we flip a fair coin and if it comes up Heads, then we stop according to the stopping rule $\tau_{U}$, while if it comes up Tails, we stop according to the stopping rule $\tau_{V}$. Hence $\tau$ is a stopping rule with respect to the enlarged filtration $\overline{\mathscr{F}}_{t}$ that consists of everything in $\mathscr{F}$ plus the two possible outcomes of the random experiment of tossing the fair coin. That is, $\overline{\mathscr{F}}_{t}=\mathscr{F}_{t} \cup\{\{$ Heads $\},\{$ Tails $\}$.

We then find the best classical 2-CUSUM stopping rule for any given value of the mean time between false alarms. This is achieved through showing the existence and uniqueness of a rule in the class of classical 2-CUSUM stopping rules that is adapted to the original filtration $\mathscr{F}_{t}$ and satisfies $J_{1}(\tau)=J_{2}(\tau)$. In particular, we point out that in the symmetric case any harmonic mean classical 2-CUSUM stopping rule satisfies $J_{1}(\tau)=J_{2}(\tau)$ and is thus the best in the classical 2-CUSUM class (and it is unique for any given mean rate between false alarms $T$ ), while in the nonsymmetric case, it is seen that there exists a unique (for any given mean rate between false alarms $T$ ) nonharmonic mean classical 2-CUSUM stopping rule that satisfies $J_{1}(\tau)=J_{2}(\tau)$ and is thus the best in the classical 2-CUSUM stopping rule class. Moreover, the class of optimal classical 2-CUSUM stopping rule is specified. This is achieved through the derivation of an expression for the first moment of a general nonharmonic mean 2-CUSUM stopping rule and its rate of change as the threshold parameter of one of its one sided CUSUM stopping branches changes. We also derive upper and lower bounds to the aforementioned moment that enhance the existing bounds of [3]. It is important to point out here that although for any harmonic mean 2-CUSUM stopping rule, the Laplace transform of its probability density function has been computed (see [12]), only a bound on the first moment of a general 2-CUSUM stopping rule is known (see [3]). We proceed with some definitions.

D e f in ition 1. Let $\nu_{1}>0$ and $\nu_{2}>0$. Define

1) $u_{t}^{+}=\left(\ln \frac{d \mathbf{P}_{0}^{1}}{d \mathbf{P}_{\infty}} \mid \mathscr{F}_{t}\right) / \mu_{1}=\xi_{t}-\frac{1}{2} \mu_{1} t ; m_{t}^{+}=\inf _{s \leqslant t} u_{s}^{+}, y_{t}^{+}=u_{t}^{+}-m_{t}^{+}$; $\tau_{1}\left(\nu_{1}\right)=\inf \left\{t>0 ; y_{t}^{+} \geqslant \nu_{1}\right\}$, and

2) $u_{t}^{-}=\ln \left(\frac{d \mathbf{P}_{0}^{2}}{d \mathbf{P}_{\infty}} \mid \mathscr{F}_{t}\right) / \mu_{2}=-\xi_{t}-\frac{1}{2} \mu_{2} t ; m_{t}^{-}=\inf _{s \in t} u_{s}^{-} ; y_{t}^{-}=u_{t}^{-}-m_{t}^{-}$; $\tau_{2}\left(\nu_{2}\right)=\inf \left\{t>0 ; y_{t}^{-} \geqslant \nu_{2}\right\}$.

The classical 2-CUSUM stopping rules are then of the form $\tau\left(\nu_{1}, \nu_{2}\right)=$ $\tau_{1}\left(\nu_{1}\right) \wedge \tau_{2}\left(\nu_{2}\right)$

We now classify 2-CUSUM rules according to the class $\mathscr{G}=$ $\left\{\tau\left(\nu_{1}, \nu_{2}\right) ; \quad \nu_{1}=\nu_{2}\right\}$ of harmonic mean rules and the classes $\mathscr{C}_{i}=$ 
$\left\{\tau\left(\nu_{i}, \nu_{j}\right) \mid \nu_{i}>\nu_{j}>0, i \neq j\right\}$ of nonharmonic mean rules. We also define the following quantities, the use of which will become apparent later.

D ef in ition 2. For $a>0$ and $b>0$, we define

1) $U^{+}(a)=\inf \left\{t>0 ; u_{t}^{+} \geqslant a\right\}, U^{-}(b)=\inf \left\{t>0 ;-u_{t}^{-} \leqslant-b\right\}$, and $\Pi(a, b)=\mathbf{P}\left(U^{+}(a)<U^{-}(b)\right)$,

2) $U_{0}^{+}(a)=\inf \left\{t>0 ; u_{t}^{+}+\frac{1}{2}\left(\mu_{1}+\mu_{2}\right) t \geqslant a\right\}, U_{0}^{-}(b)=\inf \{t>0$; $\left.-u_{t}^{-}-\frac{1}{2}\left(\mu_{1}+\mu_{2}\right) t \leqslant-b\right\}$,

3) $u_{t-}^{-}=u_{t}^{-}+\frac{1}{2}\left(\mu_{1}+\mu_{2}\right) t ; m_{t-}^{-}=\inf _{s \leqslant t} u_{s_{-}^{-}} ; y_{t-}^{-}=u_{t-}-m_{t-}$ and $\tau_{2}^{-}\left(\nu_{2}\right)=\inf \left\{t>0 ; y_{t-}^{-} \geqslant \nu_{2}\right\}$.

In the sequel we will repeatedly use the indices $i, j \in\{1,2\}$.

Definition 3 . Let $\tau \in \mathscr{C}_{i}$. Define

1) $m=\min \left\{\nu_{1}, \nu_{2}\right\}$ and $M=\max \left\{\nu_{1}, \nu_{2}\right\}$,

2) $C_{m}(x, y)=f_{m}(x)^{2} /\left(f_{m}(x)+f_{m}(y)\right), \lambda_{x}(y)=\left(y f_{x}(y)+x\right)^{-1}$, and $f_{x}(y)=\left(e^{y x}-y x-1\right) / y^{2}$,

3) $R_{\infty}^{i}=d \mathbf{E}_{\infty}[\tau] / d M, R_{j}^{i}=d \mathbf{E}_{0}^{j}[\tau] / d M$.

According to [5] and [9], we also have

$$
\begin{aligned}
\mathbf{E}_{\infty}\left[\tau_{i}\left(\nu_{i}\right)\right] & =2 f_{\nu_{i}}\left(\mu_{i}\right), \quad i=1,2, \\
\mathbf{E}_{0}^{i}\left[\tau_{i}\left(\nu_{i}\right)\right] & =2 f_{\nu_{i}}\left(-\mu_{i}\right), \quad i=1,2, \\
\mathbf{E}_{0}^{i}\left[\tau_{j}\left(\nu_{j}\right)\right] & =2 f_{\nu_{j}}\left(\mu_{j}+2 \mu_{i}\right), \quad i \neq j, \quad i, j \in\{1,2\} .
\end{aligned}
$$

For any 2-CUSUM stopping rule $\tau$ it is true (see [5]) that $J_{L}(\tau)=$ $\max \left\{\mathbf{E}_{0}^{1}[\tau], \mathbf{E}_{0}^{2}[\tau]\right\}$.

In Section 2, we derive two expressions for the first moment of a general 2-CUSUM stopping rule and an expression for the rate of change of this first moment with respect to one of the threshold parameters. In Section 3, we establish explicit upper and lower bounds on the expected value of a general 2-CUSUM stopping rule and on its rate of change with respect to one of the threshold parameters. In Section 4, we prove the uniqueness of the best 2-CUSUM stopping rule, both in the case of a symmetric change and in the case of a nonsymmetric change and specify its class. Finally, in Section 5, we conclude with some closing remarks.

2. The first moment of a general 2-CUSUM rule. We begin with our main expression for the first moment of a general 2-CUSUM stopping rule.

Theorem 1. Let $\tau\left(\nu_{1}, \nu_{2}\right)=\tau_{1}\left(\nu_{1}\right) \wedge \tau_{2}\left(\nu_{2}\right)$ be any 2-CUSUM stopping rule and denote $\tau\left(\nu_{1}, \nu_{2}\right)$ by $\tau$. Then, under any of the measures $\mathbf{P}_{\infty}, \mathbf{P}_{0}^{1}$, and $\mathbf{P}_{0}^{2}$, we have

$$
\begin{aligned}
\tau \in \mathscr{C}_{1} \Longrightarrow \mathbf{E}[\tau]=\mathbf{E}\left[\tau_{2}(m)\right][ & 1-\frac{\mathbf{E}\left[\tau_{2}(m)\right]}{\mathbf{E}\left[\tau_{1}(m)\right]+\mathbf{E}\left[\tau_{2}(m)\right]} \\
& \left.\times \lim _{n \rightarrow \infty} \Pi\left(\frac{M-m}{n}, m\right)^{n}\right],
\end{aligned}
$$




$$
\begin{aligned}
\tau \in \mathscr{C}_{2} \Longrightarrow \mathbf{E}[\tau]=\mathbf{E}\left[\tau_{1}(m)\right][ & -\frac{\mathbf{E}\left[\tau_{1}(m)\right]}{\mathbf{E}\left[\tau_{2}(m)\right]+\mathbf{E}\left[\tau_{1}(m)\right]} \\
& \left.\times \lim _{n \rightarrow \infty}\left[1-\Pi\left(m, \frac{M-m}{n}\right)\right]^{n}\right] .
\end{aligned}
$$

We notice that for any $\tau_{\mathscr{G}},(6)$ and (7) reduce to the well-known harmonic mean rule. In the following corollary we provide an alternative expression for the expected value of a general 2-CUSUM stopping rule $\tau$ through which it can be seen to be an infinitely continuously differentiable function of the thresholds $\nu_{1}$ and $\nu_{2}$ as well as the parameters $\mu_{1}$ and $\mu_{2}$.

We point out that the probability $\Pi(a, b)$, involved in the above theorem, is given in closed form in [1]. However, in our paper we focus on the derivation of easy to handle explicit expressions that lead us to the proof of the existence and uniqueness of the best 2-CUSUM stopping rule as well as the specification of its class both in the symmetric and the nonsymmetric case.

Corollary 1. Let $\tau\left(\nu_{1}, \nu_{2}\right)=\tau_{1}\left(\nu_{1}\right) \wedge \tau_{2}\left(\nu_{2}\right)$ be any 2-CUSUM stopping rule and denote $\tau\left(\nu_{1}, \nu_{2}\right)$ by $\tau$. Let $x_{\infty}^{i}, x_{1}^{i}$, and $x_{2}^{i}$ be some constants such that $x_{\infty}^{i} \in\left(-\mu_{i}, \mu_{j}\right), x_{i}^{i} \in\left(\mu_{i}, \mu_{j}+2 \mu_{i}\right), x_{j}^{i} \in\left(-\left(\mu_{i}+2 \mu_{j}\right),-\mu_{j}\right)$ for $i \neq j$. Then, for all $\tau \in \mathscr{C}_{i}, i \neq j$, we have

$$
\begin{aligned}
\mathbf{E}_{\infty}[\tau] & =2 f_{m}\left(\mu_{j}\right)\left[1-\frac{C_{m}\left(\mu_{j}, \mu_{i}\right)^{\prime}}{f_{m}\left(\mu_{j}\right)} e^{-\lambda_{m}\left(x_{\infty}^{i}\right)(M-m)}\right], \\
\mathbf{E}_{0}^{i}[\tau] & =2 f_{m}\left(\mu_{j}+2 \mu_{i}\right)\left[1-\frac{C_{m}\left(\mu_{j}+2 \mu_{i},-\mu_{i}\right)}{f_{m}\left(\mu_{j}+2 \mu_{i}\right)} e^{-\lambda_{m}\left(x_{i}^{i}\right)(M-m)}\right], \\
\mathbf{E}_{0}^{j}[\tau] & =2 f_{m}\left(-\mu_{j}\right)\left[1-\frac{C_{m}\left(-\mu_{j}, \mu_{i}+2 \mu_{j}\right)}{f_{m}\left(-\mu_{j}\right)} e^{-\lambda_{m}\left(x_{j}^{i}\right)(M-m)}\right] .
\end{aligned}
$$

The proof of this corollary will be completed in the following section after the proof of Theorem 1 and the display of all auxiliary lemmas. We now proceed to express the rate of change of the first moment of a general 2-CUSUM stopping rule with respect to $M=\max \left\{\nu_{1}, \nu_{2}\right\}$.

Corollary 2. Let $\tau\left(\nu_{1}, \nu_{2}\right)=\tau_{1}\left(\nu_{1}\right) \wedge \tau_{2}\left(\nu_{2}\right)$ be any 2-CUSUM stopping rule and denote $\tau\left(\nu_{1}, \nu_{2}\right)$ by $\tau$. Then, for all $\tau \in \mathscr{C}_{i}, i \neq j$

$$
\begin{aligned}
R_{\infty}^{i} & =C_{m}\left(\mu_{j}, \mu_{i}\right) \lambda_{m}\left(x_{\infty}^{i}\right) e^{-\lambda_{m}\left(x_{\infty}^{i}\right)(M-m)}, \\
R_{i}^{i} & =C_{m}\left(\mu_{j}+2 \mu_{i},-\mu_{i}\right) \lambda_{m}\left(x_{i}^{i}\right) e^{-\lambda_{m}\left(x_{i}^{i}\right)(M-m)}, \\
R_{j}^{i} & =C_{m}\left(-\mu_{j}, \mu_{i}+2 \mu_{j}\right) \lambda_{m}\left(x_{j}^{i}\right) e^{-\lambda_{m}\left(x_{j}^{i}\right)(M-m)},
\end{aligned}
$$

with $x_{\infty}^{i}, x_{i}^{i}, x_{j}^{i}$ as in Corollary 1 .

$\mathrm{Pr}$ o of of $\mathrm{C}$ or oll a r y 2. The result follows by considering the ratio $\lim _{\varepsilon \rightarrow 0} \varepsilon^{-1}\left(\mathbf{E}\left[\tau\left(\nu_{1}+\varepsilon, \nu_{2}\right)\right]-\mathbf{E}\left[\tau\left(\nu_{1}, \nu_{2}\right)\right]\right)$ under each of the measures $\mathbf{P}_{\infty}, \mathbf{P}_{0}^{1}$, and $\mathbf{P}_{0}^{2}$ and using Corollary 1 .

In order to prove Theorem 1 we will need to make use of two preliminary results that are summarized in the following two lemmas (see [5]). 
Lemma 1. We have

$$
\sup _{s \leqslant t}\left(y_{s}^{+}+y_{s}^{-}\right)=\max \left\{\sup _{s \leqslant t} y_{s}^{+}, \sup _{s \leqslant t} y_{s}^{-}\right\} .
$$

P r o of. Observe that $y_{t}^{+}+y_{t}^{-}=-\frac{1}{2}\left(\mu_{1}+\mu_{2}\right) t-m_{t}^{+}-m_{t}^{-}$. We notice that the process $y_{t}^{+}+y_{t}^{-}$can increase only when either $u_{t}^{+}=m_{t}^{+}$ or $u_{t}^{-}=m_{t}^{-}$, both of which cannot happen at the same time, since that would imply that $y_{t}^{+}+y_{t}^{-}$is 0 . Therefore, $y_{t}^{+}+y_{t}^{-}$is a strictly decreasing function of time unless either $y_{t}^{+}=0$ or $y_{t}^{-}=0$, at which instant $\max \left\{y_{t}^{+}, y_{t}^{-}\right\}=$ $\sup _{s \in[0, t]}\left\{\max \left\{y_{s}^{+}, y_{s}^{-}\right\}\right\}$.

As a consequence of Lemma 1, we have that

$$
\begin{aligned}
& \nu_{2} \geqslant \nu_{1} \Longrightarrow\left\{\tau_{2}<\tau_{1}\right\} \subseteq\left\{y_{\tau_{2}}^{+}=0\right\} \\
& \nu_{1} \geqslant \nu_{2} \Longrightarrow\left\{\tau_{1}<\tau_{2}\right\} \subseteq\left\{y_{\tau_{1}}^{-}=0\right\} .
\end{aligned}
$$

Lemma 2. Let $\tau_{1}$ and $\tau_{2}$ be the one-sided CUSUM stopping branches of $\tau_{\mathscr{G}} \in \mathscr{G}$ having the same threshold parameter $\nu$. We then have

$$
\mathbf{P}\left(\tau_{2}<\tau_{1}\right)=\frac{\mathbf{E}\left[\tau_{1}\right]}{\mathbf{E}\left[\tau_{1}\right]+\mathbf{E}\left[\tau_{2}\right]} \quad \text { and } \quad \mathbf{P}\left(\tau_{1}<\tau_{2}\right)=\frac{\mathbf{E}\left[\tau_{2}\right]}{\mathbf{E}\left[\tau_{1}\right]+\mathbf{E}\left[\tau_{2}\right]}
$$

$\mathrm{P}$ r o o f. For simplicity in this proof we will use $\tau$ to denote $\tau_{\mathscr{G}}$. We have $\tau=\tau_{1} \wedge \tau_{2}$. Hence, $\tau_{1}=\tau+\left(\tau_{1}-\tau\right)^{+}, \tau_{2}=\tau+\left(\tau_{2}-\tau\right)^{+}$. Conditioning on $\left\{\tau=\tau_{2}\right\}$, and subsequently on its complement, and taking expectations we have

$$
\begin{aligned}
& \mathbf{E}\left[\tau_{1}\right]=\mathbf{E}[\tau]+\mathbf{E}\left[\tau_{1}-\tau_{2} \mid \tau_{2}<\tau_{1}\right] \mathbf{P}\left(\tau_{2}<\tau_{1}\right), \\
& \mathbf{E}\left[\tau_{2}\right]=\mathbf{E}[\tau]+\mathbf{E}\left[\tau_{2}-\tau_{1} \mid \tau_{1}<\tau_{2}\right] \mathbf{P}\left(\tau_{1}<\tau_{2}\right) .
\end{aligned}
$$

Since $\tau_{1}$ and $\tau_{2}$ have the same threshold $\nu$, using (14) and (15), we obtain

$$
\left\{\tau_{2}<\tau_{1}\right\} \subseteq\left\{y_{\tau_{2}}^{+}=0\right\} \text { and }\left\{\tau_{1}<\tau_{2}\right\} \subseteq\left\{y_{\tau_{1}}^{-}=0\right\} .
$$

Therefore, (16) and (17) become

$$
\mathbf{E}\left[\tau_{1}\right]=\mathbf{E}[\tau]+\mathbf{E}\left[\tau_{1}\right] \mathbf{P}\left(\tau_{2}<\tau_{1}\right) \quad \text { and } \quad \mathbf{E}\left[\tau_{2}\right]=\mathbf{E}[\tau]+\mathbf{E}\left[\tau_{2}\right] \mathbf{P}\left(\tau_{1}<\tau_{2}\right)
$$

from which, by eliminating $\mathbf{E}[\tau]$, the result follows.

P r o of of The or e m 1. Suppose that $\nu_{1}>\nu_{2}$. Then, using (15) and (17), we have that $\mathbf{E}\left[\tau_{2}\left(\nu_{2}\right)\right]=\mathbf{E}[\tau]+\mathbf{E}\left[\tau_{2}\left(\nu_{2}\right)\right] \mathbf{P}\left(\tau_{1}\left(\nu_{1}\right)<\tau_{2}\left(\nu_{2}\right)\right)$. Hence,

$$
\mathbf{E}[\tau]=\mathbf{E}\left[\tau_{1}\left(\nu_{1}\right)\right] \mathbf{P}\left(\tau_{2}\left(\nu_{2}\right)<\tau_{1}\left(\nu_{1}\right)\right) .
$$

We now proceed to express $\mathbf{P}\left(\tau_{2}\left(\nu_{2}\right)<\tau_{1}\left(\nu_{1}\right)\right)$ in terms of $\Pi(a, b)$ as it appears in Definition 2. Notice that we can rewrite $\mathbf{P}\left(\tau_{1}\left(\nu_{1}\right)<\tau_{2}\left(\nu_{2}\right)\right)$ as

$$
\begin{aligned}
\mathbf{P}\left(\tau_{1}\left(\nu_{1}\right)<\tau_{2}\left(\nu_{2}\right)\right)= & \mathbf{P}\left(\tau_{1}\left(\nu_{1}\right)<\tau_{2}\left(\nu_{2}\right) \mid \tau_{1}\left(\nu_{2}\right)<\tau_{2}\left(\nu_{2}\right)\right) \\
& \times \mathbf{P}\left(\tau_{1}\left(\nu_{2}\right)<\tau_{2}\left(\nu_{2}\right)\right) .
\end{aligned}
$$


Using Lemma 2, however, with $\nu=\nu_{2}$, we obtain

$$
\mathbf{P}\left(\tau_{1}\left(\nu_{2}\right)<\tau_{2}\left(\nu_{2}\right)\right)=\frac{\mathbf{E}\left[\tau_{2}\left(\nu_{2}\right)\right]}{\mathbf{E}\left[\tau_{1}\left(\nu_{2}\right)\right]+\mathbf{E}\left[\tau_{2}\left(\nu_{2}\right)\right]} .
$$

To get an expression for $\mathbf{P}\left(\tau_{1}\left(\nu_{1}\right)<\tau_{2}\left(\nu_{2}\right) \mid \tau_{1}\left(\nu_{2}\right)<\tau_{2}\left(\nu_{2}\right)\right)$, we first consider the dynamics of $y_{t}^{+}$and $y_{t}^{-}$under all relevant measures. Using Definition 1 , we can write $y_{t}^{+}=u_{t}^{+}-m_{t}^{+}$and $y_{t}^{-}=u_{t}^{-}-m_{t}^{-}$, where the dynamics of $u_{t}^{+}$ and $-u_{t}^{-}$are summarized in Table 1.

Table 1. The dynamics of $u_{t}^{+}$and $-u_{t}^{-}$under the different regimes

\begin{tabular}{|l|l|l|l|}
\hline & no change $\left(\mathbf{P}_{\infty}\right)$ & change is $\mu_{1}\left(\mathbf{P}_{0}^{1}\right)$ & change is $-\mu_{2}\left(\mathbf{P}_{0}^{2}\right)$ \\
\hline$u_{t}^{+}$ & $w_{t}-\frac{1}{2} \mu_{1} t$ & $w_{t}+\frac{1}{2} \mu_{1} t$ & $w_{t}-\frac{1}{2}\left(\mu_{1}+2 \mu_{2}\right) t$ \\
\hline$-u_{t}^{-}$ & $w_{t}+\frac{1}{2} \mu_{2} t$ & $w_{t}+\frac{1}{2}\left(\mu_{2}+2 \mu_{1}\right) t$ & $w_{t}-\frac{1}{2} \mu_{2} t$ \\
\hline
\end{tabular}

Therefore, if we were to divide the interval $\left[0, \nu_{1}-\nu_{2}\right]$ into $n$ equal length intervals, then

$$
\mathbf{P}\left(\tau_{1}\left(\nu_{1}\right)<\tau_{2}\left(\nu_{2}\right) \mid \tau_{1}\left(\nu_{2}\right)<\tau_{2}\left(\nu_{2}\right)\right)=\lim _{n \rightarrow \infty} \mathbf{P}\left(\bigcap_{i=1}^{n} A_{i}\right),
$$

where $A_{i}$ is the event that $\left\{\sup _{t \in I_{i}} y_{t}^{-}<\nu_{2}\right\}$ conditioned upon the initial value of $y_{t}^{-}$at the left endpoint of each interval $I_{i}$ being equal to 0 and $\left\{I_{i}\right\}$ are the random intervals

$$
I_{i}=\left[\tau_{1}\left(\nu_{2}+(i-1) \frac{\nu_{1}-\nu_{2}}{n}\right), \tau_{1}\left(\nu_{2}+i \frac{\nu_{1}-\nu_{2}}{n}\right)\right] .
$$

Due to the strong Markov property of Brownian motion the events $\left\{A_{i}\right\}$ are independent, equiprobable and they have the same probability as the event

$$
\left\{\begin{array}{c}
\text { The process } u_{t}^{+} \text {increases by at least } n^{-1}\left(\nu_{1}-\nu_{2}\right) \text { units } \\
\text { before the process }-u_{t}^{-} \text {falls by } \nu_{2} \text { (or more) units }
\end{array}\right\} \text {. }
$$

Therefore, in view of Definition 2 and (22), identity (21) becomes

$$
\begin{aligned}
\mathbf{P}\left(\tau_{1}\left(\nu_{1}\right)<\tau_{2}\left(\nu_{2}\right) \mid \tau_{1}\left(\nu_{2}\right)<\tau_{2}\left(\nu_{2}\right)\right) & =\lim _{n \rightarrow \infty}\left[\mathbf{P}\left(A_{1}\right)\right]^{n} \\
& =\lim _{n \rightarrow \infty} \Pi\left(\frac{\nu_{1}-\nu_{2}}{n}, \nu_{2}\right)^{n} .
\end{aligned}
$$

Using equations (18)-(20) and (23) the result follows.

3. Upper and lower bounds. In this section we establish upper and lower bounds on the first moment of a general 2-CUSUM stopping rule and on its rate of change with respect to $M=\max \left\{\nu_{1}, \nu_{2}\right\}$. 
Corollary 3. Let $A_{m}^{M}(z, y, x)=2 f_{m}(z)\left[1-\left(C_{m}(z, y) / f_{m}(z)\right) e^{-x(M-m)}\right]$ and let $\tau\left(\nu_{1}, \nu_{2}\right)=\tau_{1}\left(\nu_{1}\right) \wedge \tau_{2}\left(\nu_{2}\right)$ be any 2-CUSUM stopping rule. Denote $\tau\left(\nu_{1}, \nu_{2}\right)$ by $\tau$. Then, for all $\tau \in \mathscr{C}_{i}, i \neq j$, we have

$$
\begin{aligned}
& A_{m}^{M}\left(\mu_{j}, \mu_{i}, \lambda_{m}\left(\mu_{j}\right)\right) \leqslant \mathbf{E}_{\infty}[\tau] \leqslant A_{m}^{M}\left(\mu_{j}, \mu_{i}, \lambda_{m}\left(-\mu_{i}\right)\right), \\
& A_{m}^{M}\left(\mu_{j}+2 \mu_{i},-\mu_{i}, \lambda_{m}\left(\mu_{j}+2 \mu_{i}\right)\right) \\
& \leqslant \mathbf{E}_{0}^{i}[\tau] \leqslant A_{m}^{M}\left(\mu_{j}+2 \mu_{i},-\mu_{i}, \lambda_{m}\left(\mu_{i}\right)\right), \\
& A_{m}^{M}\left(-\mu_{j}, \mu_{i}+2 \mu_{j}, \lambda_{m}\left(-\mu_{j}\right)\right) \\
& \leqslant \mathbf{E}_{0}^{j}[\tau] \leqslant A_{m}^{M}\left(-\mu_{j}, \mu_{i}+2 \mu_{j}, \lambda_{m}\left(-\left(\mu_{i}+2 \mu_{j}\right)\right)\right) .
\end{aligned}
$$

P r o of. We will prove the result for the case in which $\tau \in \mathscr{C}_{1}$, since the proof is similar in the case in which $\tau \in \mathscr{C}_{2}$. We begin by summarizing the dynamics of the processes $u_{t}^{+}+\frac{1}{2}\left(\mu_{1}+\mu_{2}\right) t$ and $-u_{t}^{-}-\frac{1}{2}\left(\mu_{1}+\mu_{2}\right) t$ as they appear in Definition 1, in Table 2.

Table 2. The dynamics of $u_{t}^{+}+\frac{1}{2}\left(\mu_{1}+\mu_{2}\right) t$ and $-u_{t}^{-}-\frac{1}{2}\left(\mu_{1}+\mu_{2}\right) t$ under the different regimes

\begin{tabular}{|l|l|l|l|}
\hline & no change $\left(\mathbf{P}_{\infty}\right)$ & change is $\mu_{1}\left(\mathbf{P}_{0}^{1}\right)$ & change is - $\mu_{2}\left(\mathbf{P}_{0}^{2}\right)$ \\
\hline$u_{t}^{+}+\frac{1}{2}\left(\mu_{1}+\mu_{2}\right) t$ & $w_{t}+\frac{1}{2} \mu_{2} t$ & $w_{t}+\frac{1}{2}\left(\mu_{2}+2 \mu_{1}\right) t$ & $w_{t}-\frac{1}{2} \mu_{2} t$ \\
\hline$-u_{t}^{-}-\frac{1}{2}\left(\mu_{1}+\mu_{2}\right) t$ & $w_{t}-\frac{1}{2} \mu_{1} t$ & $w_{t}+\frac{1}{2} \mu_{1} t$ & $w_{t}-\frac{1}{2}\left(\mu_{1}+2 \mu_{2}\right) t$ \\
\hline
\end{tabular}

From Definition 2 it is evident that under any of the measures $\mathbf{P}_{\infty}, \mathbf{P}_{0}^{1}$, and $\mathbf{P}_{0}^{2}$, the following relationships hold:

$$
\mathbf{P}\left(U^{+}(a)<U_{0}^{-}(b)\right)<\mathbf{P}\left(U^{+}(a)<U^{-}(b)\right)<\mathbf{P}\left(U_{0}^{+}(a)<U^{-}(b)\right) .
$$

Using Theorem 1 and inequalities (27), we can obtain the following upper and lower bounds on $\mathbf{E}[\tau]$ :

$$
\begin{aligned}
\mathbf{E}[\tau] & \leqslant \mathbf{E}\left[\tau_{2}\left(\nu_{2}\right)\right] \\
& \times\left[1-\frac{\mathbf{E}\left[\tau_{2}\left(\nu_{2}\right)\right]}{\mathbf{E}\left[\tau_{1}\left(\nu_{2}\right)\right]+\mathbf{E}\left[\tau_{2}\left(\nu_{2}\right)\right]} \lim _{n \rightarrow \infty} \mathbf{P}\left(U^{+}\left(\frac{\nu_{1}-\nu_{2}}{n}\right)<U_{0}^{-}\left(\nu_{2}\right)\right)^{n}\right],
\end{aligned}
$$

$\mathbf{E}[\tau] \geqslant \mathbf{E}\left[\tau_{2}\left(\nu_{2}\right)\right]$

$$
\times\left[1-\frac{\mathbf{E}\left[\tau_{2}\left(\nu_{2}\right)\right]}{\mathbf{E}\left[\tau_{1}\left(\nu_{2}\right)\right]+\mathbf{E}\left[\tau_{2}\left(\nu_{2}\right)\right]} \lim _{n \rightarrow \infty} \mathbf{P}\left(U_{0}^{+}\left(\frac{\nu_{1}-\nu_{2}}{n}\right)<U^{-}\left(\nu_{2}\right)\right)^{n}\right] .
$$

But

$$
\begin{gathered}
\lim _{n \rightarrow \infty} \mathbf{P}\left(U^{+}\left(\frac{\nu_{1}-\nu_{2}}{n}\right)<U_{0}^{-}\left(\nu_{2}\right)\right)^{n}=\mathbf{P}\left(\sup _{s \leqslant \tau_{2}^{-}\left(\nu_{2}\right)} u_{s}^{+}>\nu_{1}-\nu_{2}\right), \\
\lim _{n \rightarrow \infty} \mathbf{P}\left(U_{0}^{+}\left(\frac{\nu_{1}-\nu_{2}}{n}\right)<U^{-}\left(\nu_{2}\right)\right)^{n} \\
=\mathbf{P}\left(\sup _{s \leqslant \tau_{2}\left(\nu_{2}\right)}\left(u_{s}^{+}+\frac{1}{2}\left(\mu_{1}+\mu_{2}\right) s\right)>\nu_{1}-\nu_{2}\right),
\end{gathered}
$$


where $\tau_{2}$ is as it appears in Definition 1 and $\tau_{2}^{-}$as it appears in Definition 2. According to [11] and [6], it follows that

$$
\begin{aligned}
& \sup _{s \leqslant \tau_{2}^{-}\left(\nu_{2}\right)} u_{s}^{+} \sim \operatorname{Exponential}\left(\lambda_{\nu_{2}}(2 x)\right), \\
& \sup _{s \leqslant \tau_{2}\left(\nu_{2}\right)}\left(u_{s}^{+}+\frac{1}{2}\left(\mu_{1}+\mu_{2}\right) s\right) \sim \operatorname{Exponential}\left(\lambda_{\nu_{2}}\left(2\left(x+\frac{1}{2}\left(\mu_{1}+\mu_{2}\right)\right)\right)\right),
\end{aligned}
$$

where $x$ is the drift of the drifting Brownian motion process $u_{t}^{+}$summarized in Table 3.

Table 3. The drift of the $u_{t}^{+}$process under the different regimes

\begin{tabular}{|c|c|c|c|}
\hline & no change $\left(\mathbf{P}_{\infty}\right)$ & change is $\mu_{1}\left(\mathbf{P}_{0}^{1}\right)$ & change is $-\mu_{2}\left(\mathbf{P}_{0}^{2}\right)$ \\
\hline$x$ & $-\frac{1}{2} \mu_{1}$ & $\frac{1}{2} \mu_{1}$ & $-\frac{1}{2}\left(\mu_{1}+2 \mu_{2}\right)$ \\
\hline
\end{tabular}

Using (32) and (33) to evaluate (30) and (31) and inequalities (27) to express (28) and (29), the upper and lower bound inequalities (24), (25), and (26) follow.

Using the proof of Corollary 3 , it is now easy to prove Corollary 1.

$\mathrm{Pr}$ o o f of $\mathrm{C}$ or o ll a r y 1 . Again it suffices to show the result for the case in which $\tau \in \mathscr{C}_{1}$. Of course, it also suffices to prove the result for any one of the measures $\mathbf{P}_{\infty}, \mathbf{P}_{0}^{1}$ or $\mathbf{P}_{0}^{2}$, since the proof is similar for any other measure. To this effect, consider the function

$$
F(x)=e^{-\lambda_{\nu_{2}}(x)\left(\nu_{1}-\nu_{2}\right)}-\lim _{n \rightarrow \infty}\left[\mathbf{P}_{\infty}\left(U^{+}\left(\frac{\nu_{1}-\nu_{2}}{n}\right)<U^{-}\left(\nu_{2}\right)\right)\right]^{n} .
$$

Using (24), it follows that $F\left(\mu_{2}\right)>0$, while $F\left(-\mu_{1}\right)<0$. Therefore, by the continuity of $F(\cdot)$, it follows that there exists an $x_{\infty}^{1}$ in the interval $\left(-\mu_{1}, \mu_{2}\right)$ such that $F\left(x_{\infty}^{1}\right)=0$. That is,

$$
\lim _{n \rightarrow \infty}\left[\mathbf{P}_{\infty}\left(U^{+}\left(\frac{\nu_{1}-\nu_{2}}{n}\right)<U^{-}\left(\nu_{2}\right)\right)\right]^{n}=\exp \left\{-\lambda_{\nu_{2}}\left(x_{\infty}^{1}\right)\left(\nu_{1}-\nu_{2}\right)\right\},
$$

for some $x_{\infty}^{1} \in\left(-\mu_{1}, \mu_{2}\right)$. Substituting the above expression into the expression for $\mathbf{E}[\tau]$ given in Theorem 1, the result follows.

$\mathrm{R}$ e $\mathrm{m}$ a r k 3. By looking at Table 1, we notice that

1) $u_{t}^{+}\left(\mathbf{P}_{0}^{1}\right)-u_{t}^{+}\left(\mathbf{P}_{\infty}\right)=-u_{t}^{-}\left(\mathbf{P}_{0}^{1}\right)+u_{t}^{-}\left(\mathbf{P}_{\infty}\right)=\mu_{1} t$,

2) $u_{t}^{+}\left(\mathbf{P}_{\infty}\right)-u_{t}^{+}\left(\mathbf{P}_{0}^{2}\right)=-u_{t}^{-}\left(\mathbf{P}_{\infty}\right)+u_{t}^{-}\left(\mathbf{P}_{0}^{2}\right)=\mu_{2} t$.

Therefore, the effect of changing the measure from $\mathbf{P}_{\infty}$ to $\mathbf{P}_{0}^{1}$ is to add the drift $\mu_{1}$ to both the processes $u_{t}^{+}$and $-u_{t}^{-}$. The effect of changing the measure from $\mathbf{P}_{0}^{2}$ to $\mathbf{P}_{\infty}$ is to add the drift $\mu_{2}$ to both of the above processes. And finally, the effect of changing the measure from $\mathbf{P}_{0}^{2}$ to $\mathbf{P}_{0}^{1}$ is to add the drift $\mu_{1}+\mu_{2}$ to the same processes. This is reflected in the argument of the function $\lambda_{\nu_{2}}(\cdot)$. From equations (32) and (33), we notice that this change of 
measure results in a shift in the argument of the function $\lambda_{\nu_{2}}(\cdot)$ by $2 \mu_{1}, 2 \mu_{2}$, and $2\left(\mu_{2}+\mu_{1}\right)$, respectively. Comparing this to expression (35) and the equivalent expressions

$$
\lim _{n \rightarrow \infty}\left[\mathbf{P}_{0}^{i}\left(U^{+}\left(\frac{\nu_{1}-\nu_{2}}{n}\right)<U^{-}\left(\nu_{2}\right)\right)\right]^{n}=\exp \left\{-\lambda_{\nu_{2}}\left(x_{i}^{1}\right)\left(\nu_{1}-\nu_{2}\right)\right\},
$$

$i=1,2$, we deduce that

$$
x_{1}^{1}-x_{\infty}^{1}=2 \mu_{1}, \quad x_{\infty}^{1}-x_{2}^{1}=2 \mu_{2}, \quad \text { and } \quad x_{1}^{1}-x_{2}^{1}=2\left(\mu_{1}+\mu_{2}\right) .
$$

(More generally, $x_{i}^{i}-x_{\infty}^{i}=2 \mu_{i}, x_{\infty}^{i}-x_{j}^{i}=2 \mu_{j}$, and $x_{i}^{i}-x_{j}^{i}=2\left(\mu_{i}+\mu_{j}\right)$ for $i \neq j$.)

We conclude this section with upper and lower bounds on the rates of change of Definition 3.

Corollary 4. Let $\tau\left(\nu_{1}, \nu_{2}\right)=\tau_{1}\left(\nu_{1}\right) \wedge \tau_{2}\left(\nu_{2}\right)$ be any 2-CUSUM stopping rule and denote $\tau\left(\nu_{1}, \nu_{2}\right)$ by $\tau$. For all $\tau \in \mathscr{C}_{i}$, let $\nu_{i}=\nu_{j}+\varepsilon$. Then, as $\varepsilon \rightarrow 0$

$$
\begin{aligned}
C_{\nu_{j}}\left(\mu_{j}, \mu_{i}\right) \lambda_{\nu_{j}}\left(\mu_{j}\right) \leqslant\left. R_{\infty}^{i}\right|_{\nu_{1}=\nu_{2}} \leqslant & C_{\nu_{j}}\left(\mu_{j}, \mu_{i}\right) \lambda_{\nu_{j}}\left(-\mu_{i}\right), \\
C_{\nu_{j}}\left(\mu_{j}+2 \mu_{i},-\mu_{i}\right) \lambda_{\nu_{j}}\left(\mu_{j}+2 \mu_{i}\right) \leqslant\left. R_{i}^{i}\right|_{\nu_{1}=\nu_{2}} \leqslant & C_{\nu_{j}}\left(\mu_{j}+2 \mu_{i},-\mu_{i}\right) \\
& \times \lambda_{\nu_{j}}\left(\mu_{i}\right), \\
C_{\nu_{j}}\left(-\mu_{j}, \mu_{i}+2 \mu_{j}\right) \lambda_{\nu_{j}}\left(-\mu_{j}\right) \leqslant\left. R_{j}^{i}\right|_{\nu_{1}=\nu_{2}} \leqslant & C_{\nu_{j}}\left(-\mu_{j}, \mu_{i}+2 \mu_{j}\right) \\
& \times \lambda_{\nu_{j}}\left(-\left(\mu_{i}+2 \mu_{j}\right)\right) .
\end{aligned}
$$

P r o o f. This corollary is an immediate consequence of Corollary 2 and the fact that the function $\lambda_{x}(y)$ is strictly decreasing in $y$.

\section{The best 2-CUSUM stopping rule.}

Lemma 3. With $R_{i}^{i}$ and $R_{j}^{i}, i \neq j$, as in Definition 3, we have that $\mu_{i} \leqslant \mu_{j} \Rightarrow R_{i}^{i}>R_{j}^{i}$.

P r o of. Let $i=1$. Using Corollary 2, we have that

$$
\begin{aligned}
& R_{1}^{1}=C_{\nu_{2}}\left(\mu_{2}+2 \mu_{1},-\mu_{1}\right) \lambda_{\nu_{2}}\left(x_{1}^{1}\right) \exp \left\{-\lambda_{\nu_{2}}\left(x_{1}\right)\left(\nu_{1}-\nu_{2}\right)\right\}, \\
& R_{2}^{1}=C_{\nu_{2}}\left(\mu_{2}, \mu_{1}\right) \lambda_{\nu_{2}}\left(x_{2}^{1}\right) \exp \left\{-\lambda_{\nu_{2}}\left(x_{2}^{1}\right)\left(\nu_{1}-\nu_{2}\right)\right\} .
\end{aligned}
$$

Since $x_{1}^{1}>x_{2}^{1}$, it follows that $\exp \left\{-\lambda_{\nu_{2}}\left(x_{1}^{1}\right)\left(\nu_{1}-\nu_{2}\right)\right\}>\exp \left\{-\lambda_{\nu_{2}}\left(x_{2}^{1}\right) \times\right.$ $\left.\left(\nu_{1}-\nu_{2}\right)\right\}$. Hence to establish the desired inequality it suffices to show that

$$
\frac{\lambda_{\nu_{2}}\left(x_{1}^{1}\right)}{\lambda_{\nu_{2}}\left(x_{2}^{1}\right)}>\frac{C_{\nu_{2}}\left(\mu_{2}, \mu_{1}\right)}{C_{\nu_{2}}\left(\mu_{2}+2 \mu_{1},-\mu_{1}\right)} .
$$

Using the fact that the function $h(x)=\left[x /\left(e^{x}-1\right)\right]\left[(x-\alpha) /\left(e^{x-\alpha}-1\right)\right]^{-1}$ for any $\alpha>0$ (and in particular for $\alpha=2\left(\mu_{1}+\mu_{2}\right)=x_{1}^{1}-x_{2}^{1}$, see (37)) is strictly decreasing for all $x$, we obtain $\lambda_{\nu_{2}}\left(x_{1}^{1}\right) / \lambda_{\nu_{2}}\left(x_{2}^{1}\right)>\lambda_{\nu_{2}}\left(\mu_{2}+2 \mu_{1}\right) / \lambda_{\nu_{2}}\left(-\mu_{2}\right)$. Therefore, to prove inequality (43) it suffices to show that

$$
C_{\nu_{2}}\left(\mu_{2}+2 \mu_{1},-\mu_{1}\right) \lambda_{\nu_{2}}\left(\mu_{2}+2 \mu_{1}\right)>C_{\nu_{2}}\left(-\mu_{2}, \mu_{1}+2 \mu_{2}\right) \lambda_{\nu_{2}}\left(-\mu_{2}\right) .
$$


But by the convexity of the function $f_{x}(y)$ in $y$, it follows that for $\mu_{1}<\mu_{2}$,

$$
f_{\nu_{2}}\left(\mu_{1}+2 \mu_{2}\right)+f_{\nu_{2}}\left(-\mu_{2}\right)>f_{\nu_{2}}\left(\mu_{2}+2 \mu_{1}\right)+f_{\nu_{2}}\left(-\mu_{1}\right),
$$

so that

$$
\left[f_{\nu_{2}}\left(\mu_{1}+2 \mu_{2}\right)+f_{\nu_{2}}\left(-\mu_{2}\right)\right]^{-1}<\left[f_{\nu_{2}}\left(\mu_{2}+2 \mu_{1}\right)+f_{\nu_{2}}\left(-\mu_{1}\right)\right]^{-1} .
$$

Hence, substituting for $C_{\nu_{2}}(\cdot, \cdot)$ and for $\lambda_{\nu_{2}}(\cdot)$ and multiplying through by $\nu_{2}$, it becomes evident that to show (44), it suffices to show that the function $[1-1 / g(x)]^{2} g(x) / x^{2}$, with $g(x)=\left(e^{x}-1\right) / x$, is strictly increasing for all $x$, which is true. Lemma 3 is proved.

Lemma 4. Let $\tau_{\mathscr{G}}$ be a 2-CUSUM harmonic mean rule with threshold parameter $\nu$. Then for $i \neq j$,

$$
\begin{aligned}
& \mu_{i}<\mu_{j} \Longleftrightarrow \mathbf{E}_{0}^{i}\left[\tau_{\mathscr{G}}\right]>\mathbf{E}_{0}^{j}\left[\tau_{\mathscr{G}}\right], \\
& \mu_{2}=\mu_{1} \Longleftrightarrow \mathbf{E}_{0}^{2}\left[\tau_{\mathscr{G}}\right]=\mathbf{E}_{0}^{1}\left[\tau_{\mathscr{G}}\right] .
\end{aligned}
$$

$\mathrm{P} \mathrm{r}$ o of. Let us begin with the case that $i=1$. In view of expressions (4), (5) and the fact that, since $\tau_{\mathscr{G}}$ is a 2-CUSUM harmonic mean rule, its expected value is the harmonic mean of the expected values of its respective one-sided CUSUM branches $\tau_{1}$ and $\tau_{2},(48)$ is trivial. Inequality (47) follows by the harmonic mean rule which implies

$$
\begin{aligned}
& \frac{1}{\mathbf{E}_{0}^{1}\left[\tau_{\mathscr{G}}\right]}=\frac{1}{2 f_{\nu}\left(\mu_{2}+2 \mu_{1}\right)}+\frac{1}{2 f_{\nu}\left(-\mu_{1}\right)}, \\
& \frac{1}{\mathbf{E}_{0}^{2}\left[\tau_{\mathscr{G}}\right]}=\frac{1}{2 f_{\nu}\left(\mu_{1}+2 \mu_{2}\right)}+\frac{1}{2 f_{\nu}\left(-\mu_{2}\right)},
\end{aligned}
$$

by the convexity of the function $1 / f_{x}(y)$ in $y$, and by the facts that $2 \mu_{2}+\mu_{1}>$ $\mu_{2}+2 \mu_{1}>-\mu_{1}>-\mu_{2}$, while $\left(2 \mu_{2}+\mu_{1}\right)-\left(\mu_{2}+2 \mu_{1}\right)=\left(-\mu_{1}\right)-\left(-\mu_{2}\right)=$ $\mu_{2}-\mu_{1}$. Similarly, for $i=2$. Lemma 4 is proved.

As a consequence of Remark 1, the best 2-CUSUM stopping rule $\tau^{*}$ will be a member of the class of equalizer 2-CUSUM stopping rules $\mathscr{E}=\{\tau \in$ 2-CUSUM class; $\left.\mathbf{E}_{0}^{1}[\tau]=\mathbf{E}_{0}^{2}[\tau]\right\}$. We proceed in this section with the main theorem that establishes not only that the class $\mathscr{E}$ is nonempty, but that in fact it has one element.

Theorem 2. The best 2-CUSUM stopping rule $\tau^{*}$ exists and is unique and we have

$$
\begin{aligned}
& \mu_{i}<\mu_{j} \Longrightarrow \tau^{*} \in \mathscr{C}_{j}, \quad i \neq j, \\
& \mu_{1}=\mu_{2} \Longrightarrow \tau^{*} \in \mathscr{G} .
\end{aligned}
$$

$\mathrm{P}$ r o o f. Let us begin with $i=1$. Using Lemma 4, we see that $\mathbf{E}_{0}^{1}\left[\tau_{\mathscr{G}}\right]>\mathbf{E}_{0}^{2}\left[\tau_{\mathscr{G}}\right]$. Lemma 4 is proved. But if we start increasing $\nu_{2}$ above $\nu_{1}$, of course, as can readily be seen from the expression for $\mathbf{E}[\tau]$ appearing in Theorem 1, both the left-hand side and the right-hand side of the above inequality will increase continuously (see Corollary 1), but the left-hand side 
will increase at the rate $R_{1}^{2}$, while the right-hand side will increase at the rate $R_{2}^{2}$. According to Lemma $3, R_{1}^{2}<R_{2}^{2}$. Therefore, there exists a unique $\nu_{2}>\nu_{1}$ such that $\mathbf{E}_{0}^{1}[\tau]=\mathbf{E}_{0}^{2}[\tau]$.

For the second case $i=2$, again using Lemma 4, we see that $\mathbf{E}_{0}^{1}\left[\tau_{\mathscr{G}}\right]<\mathbf{E}_{0}^{2}\left[\tau_{\mathscr{G}}\right]$. But if we start increasing $\nu_{1}$ above $\nu_{2}$, both the left-hand side and the right-hand side of the above inequality will increase continuously (see Corollary 1), but the left-hand side will increase at the rate $R_{1}^{1}$, while the right-hand side will increase at rate $R_{2}^{1}$. According to Lemma 3, $R_{1}^{1}>R_{2}^{1}$. Therefore, there exists a unique $\nu_{1}>\nu_{2}$ such that $\mathbf{E}_{0}^{1}[\tau]=\mathbf{E}_{0}^{2}[\tau]$.

In the third and final case we see that any $\tau \in \mathscr{G}$ is also a member of $\mathscr{E}$, that is, $\mathbf{E}_{0}^{1}\left[\tau_{\mathscr{G}}\right]=\mathbf{E}_{0}^{2}\left[\tau_{\mathscr{G}}\right]$. If we start increasing $\nu_{1}$ above $\nu_{2}$, since $R_{1}^{1}>R_{2}^{1}$, although both the left-hand side and the right-hand side of this equality will increase, the right-hand side will increase faster than the left-hand side, resulting in $\mathbf{E}_{0}^{1}[\tau]>\mathbf{E}_{0}^{2}[\tau]$. Similarly, if we start increasing $\nu_{2}$ above $\nu_{1}$, since $R_{1}^{2}<R_{2}^{2}$, although both the left-hand side and the right-hand side of this equality will increase, the right-hand side will increase faster than the left-hand side, resulting in $\mathbf{E}_{0}^{1}[\tau]<\mathbf{E}_{0}^{2}[\tau]$. Therefore, $\nu_{1}=\nu_{2}$ is the unique case for which equality will hold. This completes the proof of Theorem 2 .

5. Discussion and concluding remarks. In this paper, we have proved existence and uniqueness of the best 2-CUSUM stopping rule for any given level of the mean time between false alarms. In particular, we have shown that the best 2-CUSUM stopping rule lies in the class $\mathscr{C}_{1}$ in the case in which $\mu_{1}>\mu_{2}$ and in the class $\mathscr{C}_{2}$ in the opposite case, while it is a harmonic mean rule in the case in which $\mu_{1}=\mu_{2}$. We have been able to prove this result by deriving and using useful expressions for the first moment of a general 2-CUSUM stopping rule and its rate of change as the largest of the two thresholds changes. In fact the final proof is based on the comparison of this rate of change under the different regimes $\mathbf{P}_{0}^{1}$ and $\mathbf{P}_{0}^{2}$. It is these same expressions that also have helped us derive upper and lower bounds on the first moment and its rate of change of a general 2-CUSUM stopping rule.

A very interesting problem is to find the best 2-CUSUM stopping rule for the case in which the magnitude of the drift $\mu$ of the Brownian motion after the change point $\theta$ is unknown but satisfies either $\mu \geqslant \mu_{1}$ or $\mu \leqslant \mu_{2}$. Another interesting problem is to compare the performance of the best classical 2-CUSUM stopping rule $\tau$ that lies in the class $\mathscr{E}$ to the performance of the modified 2-CUSUM harmonic mean drift equalizer rules in the case of a nonsymmetric change in the drift of Brownian motion (see [4]). The difficulty in successfully carrying out this comparison lies in the fact that the closed form expression for the first moment of a general 2-CUSUM nonharmonic mean rule appearing in Theorem 1 involves the computation of a limit of the probability that Brownian motion hits two linear nonintersecting boundaries of different slopes (see [1]). This expression is quite involved and 
as such, this comparison could only be carried out numerically. The ultimate problem is to find the optimal solution to (2) both in the symmetric and in the nonsymmetric cases. Of course, the classical 2-CUSUM stopping rules, although not optimal, have been shown to have very strong asymptotic optimality properties as the mean time between false alarms tends to $\infty$, not only under the proposed extended Lorden performance measure (see [5]) but also under the performance measure $\sup _{\theta} \mathbf{E}[\tau-\theta \mid \tau>\theta]$ (see [10]). Therefore, specifying the best in this class is a useful contribution to a field with 50-year old open problems as the one described above.

Acknowledgment. We thank Drs. George Moustakides, Mark Brown, and Gerardo Hernandez del-Valle.

\section{СПИСОК ЛИТЕРАТУРЫ}

1. Anderson T. W. A modification of the sequential probability ratio test to reduce the sample size. - Ann. Math. Statist., 1960, v. 31, p. 165-197.

2. Beibel M. A note on Ritov's Bayes approach to the minimax property of the CUSUM procedure. - Ann. Statist., 1996, v. 24, № 4, p. 1804-1812.

3. Dragalin V.P. The design and analysis of 2-CUSUM procedure. - Comm. Statist. Simulation Comput., 1997, v. 26, № 1, p. 67-81.

4. Hadjiliadis $O$. Optimality of the 2-CUSUM drift equalizer rules for detecting twosided alternatives in the Brownian motion model. - J. Appl. Probab., 2005, v. 42, № 4, p. 1183-1193.

5. Hadjiliadis O., Moustakides G. V. Optimal and asymptotically optimal CUSUM rules for change point detection in the Brownian motion model with multiple alternatives. - Теория вероятн. и ее примен., 2005, т. 50, в. 1, p. 131-144.

6. Lehoczky J.P. Formulas for stopped diffusion processes with stopping times based on the maximum. - Ann. Probab., 1977, v. 5, № 4, p. 601-607.

7. Moustakides G. V. Optimal stopping times for detecting changes in distributions. Ann. Statist., 1986, v. 14, № 4, p. 1379-1387.

8. Ширяев A.H. Минимаксная оптимальность метода кумулятивных сумм (CUSUM) в случае непрерывного времени. - Успехи матем. наук, 1996, т. 51, № 4, c. 173-174.

9. Siegmund D. Sequential Analysis. Tests and Confidence Intervals. New York: Springer-Verlag, 1985, $272 \mathrm{p}$.

10. Тартаковский А.Г. Асимптотически минимаксное многоальтернативное последовательное правило обнаружения разладки. - Тр. МИАН, 1993, т. 202, c. $287-295$.

11. Taylor H. M. A stopped Brownian motion formula. - Ann. Probab., 1975, v. 3, p. 234-246.

12. Yashchin E. On a unified approach to the analysis of two-sided cumulative sum control schemes with headstarts. - Adv. in Appl. Probab., 1985, v. 17, p. 562-593.

Поступила в редакцию 23.V.2007 